\title{
Enhancing daily light exposure increases the antibody response to influenza vaccination in patients with dementia
}

Mirjam Münch, $\mathrm{PhD}^{1,2^{\star}}$, Rolf Goldbach, $\mathrm{MD}^{3}$, Naomi Zumstein, $\mathrm{PhD}^{4,5}$, Petra Vonmoos $\mathrm{MSc}^{6}$, Jean-Louis Scartezzini PhD ${ }^{1}$, Anna Wirz-Justice $\mathrm{PhD}^{7}$, Christian Cajochen $\mathrm{PhD}^{7}$

${ }^{*}$ corresponding author

${ }^{1}$ Solar Energy and Building Physics Laboratory, Environmental and Civil Engineering Institute, Ecole Polytechnique Fédérale de Lausanne (Switzerland); ${ }^{2}$ Sleep/Wake Research Centre, University of Wellington, Wellington New Zealand; ${ }^{3}$ Geriatric Service of the City of Zurich, Zurich (Switzerland); ${ }^{4}$ Department of Anthropology, McGill University, Montreal (Canada); 5 University Research Priority Program "Dynamics of Healthy Aging”, University of Zurich, Zurich (Switzerland); ${ }^{6}$ Sonnweid - The Home, Wetzikon (Switzerland); ${ }^{7}$ Centre for Chronobiology, Psychiatric Hospital, Transfaculty Research Platform, Molecular and Cognitive Neurosciences, University of Basel (Switzerland) 


\begin{abstract}
Enhancing lighting conditions in institutions for individuals with dementia improves their sleep, circadian rhythms and well-being. Here, we tested whether a greater long-term daily light exposure supports the immune response to the annual influenza vaccination. Eighty older institutionalised patients suffering from dementia (54 women and 26 men) continuously wore an activity tracker for 8 weeks to assess individual light exposure and restactivity cycles. The patients' immune response was analysed from two blood samples taken before and 4 - 5 weeks after the annual influenza vaccination. Individual antibody concentrations to three influenza virus strains $(\mathrm{H} 3 \mathrm{~N} 2, \mathrm{H} 1 \mathrm{~N} 1, \mathrm{IB})$ were quantified via hemagglutination inhibition assays. By quantifying individual light exposure profiles (including daylight), we classified the patients into a low and a high light exposure group based on a median illuminance of 392.6 lux. The two light exposure groups did not differ in cognitive impairment severity, age or gender distribution. However, patients in the high light exposure group showed a significantly greater circadian rest-activity amplitude (i.e. more daytime activity and less nighttime activity) along with a significantly greater antibody titer increase to the H3N2 vaccine than patients in the low light exposure group, despite similar prevaccination concentrations. Sufficient seroprotective responses to all three influenza virus strains were attained for $\geq 75 \%$ of participants. These data provide first evidence for an enhanced immune response in patients with dementia when they received more daily light. Increasing daily light exposure may have beneficial effects on the human immune system, either directly or via circadian rhythm stabilisation.
\end{abstract}




\section{Introduction}

In older patients with dementia, the decline of cognitive functions is often accompanied by disturbances in sleep-wake rhythms as well as alterations in mood, behaviour and daily activities ${ }^{1,2}$. In this context, it is well documented that the neurons of the central circadian pacemaker in the brain, the suprachiasmatic nuclei (SCN), undergo a progressive decline with dementia ${ }^{3}$, resulting in a decrease in circadian rhythm amplitude and fluctuations in circadian phase with consequently weaker entrainment to the 24-h day ${ }^{4}$. These changes in the SCN, together with the natural age-related changes in the visual system, contribute to deterioration in mood, sleep-wake cycles and behaviour, and together, these symptoms increase the need for more intensive care and medication prescription ${ }^{5}$. Clinically, exposure to bright light at the appropriate time of day improves behavioural symptoms as well as sleep-wake rhythms in older demented patients ${ }^{6-14}$ and slows down cognitive deterioration $^{12}$ (for a systematic review see ${ }^{15}$ ). Simulated dawn and dusk at the bedside of institutionalised demented patients was found to advance nocturnal sleep onset by one hour ${ }^{16}$ and improve mood and wellbeing in the morning ${ }^{17}$.

Another difficulty among institutionalised older patients are accelerating mortality factors, such as recurrent epidemics of influenza ${ }^{18}$, one of the 10 leading causes for deaths in the USA. The mortality risk can be decreased by winter flu shots ${ }^{19}$, which are recommended for older patients in long-term care institutions ${ }^{20}$. However, influenza vaccination is less effective in older than young individuals ${ }^{21}$, and the immune response is attenuated with age ${ }^{19,22}$. IgA and $\lg$ antibody concentrations decrease with age, with a faster decline of the antibody titers ${ }^{20}$, especially in very old and frail adults. In consequence, older individuals are likely to be insufficiently protected by vaccination ${ }^{23,24}$.

During the COVID-19 pandemic, the older population, especially those individuals who live in institutions (e.g. nursing homes) have been most vulnerable to infections, ${ }^{25-27}$ along with a significantly higher mortality risk when positively tested for COVID-19 ${ }^{28}$. There is a (bidirectional) role of sleep ${ }^{29,30}$ and the circadian clock on different immune functions ${ }^{31-}$ ${ }^{33}$, the SCN essentially modulating innate and adaptive immune responses (reviewed in ${ }^{34,35}$ ). Aberrant light exposure (such as with shift work or jet-lag) can induce phase shifts in many circadian clock controlled functions, including immune responses ${ }^{36,37}$. The consequences of such desynchronisation are dampened circadian amplitudes of SCN cell expression in animals ${ }^{38}$. In humans, misaligned rhythms appear to be linked to serious health problems such as higher risk for cardiovascular, metabolic and neurodegenerative disease, cancer and impaired immune function ${ }^{31}$. Thus, indirectly, decreased circadian amplitudes are likely to impair the ability to respond to infections. So far, only few studies have addressed the direct impact of ocular light exposure on the human immune system ${ }^{39}$. One study showed that 
exposure to continuous bright light during daytime (i.e. polychromatic white electric light; $5000 \mathrm{Ix}$; between 6:30am and 10:30pm) significantly increased unspecific salivary IgA antibody formation in healthy young subjects ${ }^{40}$. The authors concluded that brighter light exposure during daytime activated a greater immune response in human mucosa cells ${ }^{40}$.

Enhanced daytime light exposure can increase circadian amplitude of rest activity cycles in older, institutionalised individuals (including those suffering from dementia) ${ }^{7,13}$. It is not known whether daily exposure to bright light might also ameliorate adaptive immune responses either indirectly by increasing zeitgeber strength to stabilise the circadian system or directly via an acute action on brain areas regulating innate immune responses. One example of such an adaptive immune reaction is the production of specific antibodies in response to an influenza virus vaccination. An increased specific antibody response to vaccination after enhanced environmental lighting conditions would ameliorate virus protection and thus directly improve general health in these vulnerable patients

Thus, the aim of this study was to assess vaccine responses to three different virus stains of the annual vaccine in a cohort of institutionalised patients with severe dementia. We hypothesised that patients with higher daily light exposure over several weeks would show increased antibody titers in response to the influenza vaccination than patients with lower daily exposure.

\section{Methods and Participants}

\section{Study design}

The cross-sectional study took place during 8 weeks in fall/winter in a Nursing Home in Wetzikon (Zurich, Switzerland) in 2012. Patients in twelve different wards spent time in dayrooms equipped with conventional or 'dynamic lighting' systems, where illuminance and correlated colour temperature varied across the day ${ }^{11}$. We have previously reported the results from patients' daily activities, agitation, alertness mood, quality of life (from questionnaires assessed by staff members), rest-activity cycles, sleep (derived from activity monitors), and melatonin concentrations (from saliva samples) ${ }^{11}$. Here we present the results of the specific antibody responses to the annual influenza vaccination.

\section{Participants}

The study group comprised patients over 50 years with one of the following dementia diagnoses (according to DSM-IV): vascular dementia, Alzheimer dementia, frontotemporal dementia, Parkinson's dementia or mixed forms of dementia. Initially, 104 patients were included in the study ${ }^{11}$. Here, only patients who had worn the activity watches, received the annual flu shot, and gave two blood samples, were included in the analysis $(n=80$; see 
Supplemental Table S1 for exclusion details). None of the patients was visually blind. Mean age was $78.3 \pm 8.9$ years ( 54 women, 26 men; range 55 - 95 years). Ethical approval for all study procedures was obtained from the local Ethical Review Board (KEK, Zurich, Switzerland, protocol \# KEK-ZH 2012-0059, now SWISSMEDIC). Written informed consent for study procedures was obtained from family members or legal representatives prior to study begin.

\section{Individual light exposures}

Individual light exposure (illuminance) was recorded via wrist-worn activity monitors equipped with a calibrated light sensor (Motion Watch $8 \AA$, Camntech, UK). All recordings were downloaded weekly to a PC and visually inspected. Light and rest-activity data was scored by trained assistants (see ${ }^{11}$ for a detailed description). In brief, if there was a 24-h day with less than 3 hours of recorded rest-activity, the file was edited with the 24-hour mean of this person. If there was a gap with no rest-activity data for more than 3 hours, that 24-h period was excluded from further analyses. The same criteria were also applied for the light recordings except that if a 24-h period contained rest-activity, but no light data for more than 3 hours (due to coverage of the sensor by sleeves), only the light data was not used, and if the gap was less than 3 hours, the light data was also interpolated with the $24-\mathrm{h}$ mean of that day.

In order to create high vs. low light exposure groups (similarly to what was done for the previously reported data ${ }^{11}$ ), median illuminance across 8 weeks between 8:00 and 18:00 was calculated for each participant (426.1 \pm 304.4 lux; mean \pm SD). In a next step, a median split of these data $(n=80)$ resulted in a group with higher mean light exposure (= high light group; i.e. $>392.65 \mathrm{~lx} ; \mathrm{n}=40,27$ women, 13 men) and a group with lower mean light exposure (= low light group; i.e. < $392.65 \mathrm{Ix} ; \mathrm{n}=40,27$ women, 13 men,). The two light exposure groups did not differ in age (low light group = 79.0 \pm 9.2 years; high light group = $77.7 \pm 8.6$ years; $p=0.6$; Wilcoxon 2-sample test), or cognitive impairment, as assessed by the Severe-Mini Mental State Evaluation (S-MMSE before the flu shot ${ }^{41}$ ). The S-MMSE score was $8.1 \pm 1.5$ for the low light group and $7.8 \pm 1.5$ for the high light group $(p=0.93$ Wilcoxon 2-sample test).

\section{Rest-activity cycles and sleep}

From the wrist worn monitors, circadian rest-activity data were derived as described in ${ }^{11}$ and above. All data which included 24-h days per patient underwent a non-linear circadian regression analysis ${ }^{13,42}$, resulting in the following variables: inter-daily stability (IS), inter-daily variability (IV), and relative amplitude (RA). The RA is defined as the ratio of the 10 hours with highest activity (M10) relative to the 5 hours with lowest activity (L5) per 24 
hours. Sleep variables from night time sleep episodes were determined by the software Sleep Analysis v7.23 (Camntech UK). Bed- and wake times were assessed as described in 11 and sleep variables were re-analysed for the 80 participants: habitual bedtime, wake time, time in bed, sleep duration, sleep efficiency (ratio between sleep duration: time in bed) and fragmentation index

\section{Blood samples and influenza vaccination}

Two blood samples were obtained by standard venous puncture, one immediately before and one approximately 4 weeks after the annual influenza vaccination. A total of $8 \mathrm{ml}$ blood was drawn from each patient by professional staff members of the nursing home before noon. The blood sample was sent to an external laboratory for general analyses (Medica AG, Zürich, Switzerland; Table 1). For the specific antibody titer analysis (Prof. A-C Siegrist, University of Geneva), $4 \mathrm{ml}$ whole blood was coagulated within 1 hour after the sample was taken at room temperature, centrifuged, and the serum pipetted into tubes (Eppendorf ${ }^{\circledR}$ ) and immediately frozen at $-20{ }^{\circ} \mathrm{C}$ before sending to the University of Geneva for hemagglutination inhibition assays (HIA) ${ }^{43}$.

The vaccination was performed in week 44 (i.e. between Oct. $29^{\text {th }}$ and Nov. $4^{\text {th }}, 2012$; according to national recommendations in Switzerland), except for three patients who received the first blood sample and the flu shot in week 46 and the second blood sample at the end of week 50 (due to an acute infection in week 44). The trivalent vaccine Fluarix $₫$ (GlaxoSmithKline Biologicals, UK) was applied via intramuscular injection in the patients' upper arm by the nursing home staff. The vaccine contained attenuated virus particles against influenza A virus (H3N2, H1N1) as well as against influenza B virus (IB). The strains matched the World Health Organization (WHO) recommendations for the influenza season 2012/2013. Immune responses were calculated only in patients who received the flu shot and had no acute infection (i.e. less than 15'000 Leucocytes/ $\mu$ l) which resulted in 80 patients. Patients who had very high antibody titer concentrations (> 1000) in the pre-vaccination blood sample for one of the three virus strains were also excluded for the analysis of that virus strain, which was the case in two patients for the H3N2 antibody titer, and in one patient for the H1N1 antibody titer.

\section{Statistical analysis}

For rest-activity cycles and sleep, the same analyses as described in ${ }^{11}$ were performed for the subset of participants undergoing the antibody titer analysis $(n=80)$. For influenza vaccination, the pre- and post-vaccination antibody titers and the ratio (prevaccination/post-vaccination) were used to compare the two light exposure groups. The fixed factors LIGHT EXPOSURE GROUP (low light vs. high light), AGE as a categorical factor (on 
dichotomized variables derived from median split of age, i.e. $<80.0$ or $>79 \mathrm{y}$ ), and SEX as well as their interactions were added to the model. Subject was added as random factor. For general blood variables the repeated factor SESSION (i.e. pre- and post-vaccination blood sample) was included. Statistical comparisons were performed with a generalized linear mixed model (GLIMMX; with a lognormal distribution if the data was not normally distributed) by using the Software Package SAS (SAS Institute Inc., Cary, NC, USA; v 9.4). Degrees of freedom were determined with the Satterthwaite approximation and post-hoc comparisons were performed with the Tukey-Kramer test (corrected for multiple comparisons).

In order to compare the overall immune protection against the influenza virus, we determined geometric mean titers (GMTs) for pre- and post-vaccination values ${ }^{43}$. The differences between high and low light exposure groups for post-vaccination GMTs were compared by survival analyses (Kaplan-Meier on log-ranked values; Sigma Plot v11.0, Statsoft Software Inc). For both light exposure groups, seroprotection rates (which are defined as percentage of patients with post-vaccination antibody titer $\geq 1: 40$ ) and seroconversion rates (described in reference ${ }^{43}$ ), defined as percentage of patients with 4fold increase of pre-vaccination GMT titer, were also calculated.

\section{Results}

\section{Rest-activity cycles and sleep}

Rest-activity cycles revealed a significantly higher inter-daily stability (IS) and relative amplitude (RA) in the high light group compared to the low light group (Table 1; main effect of LIGHT EXPOSURE GROUP; $\left.p<0.05 ; n=80 ; F_{1,72}>4.2 ; p<0.05\right)$. In general, IS and RA were higher in women than men (IS: women: $0.39 \pm 0.03$, men: $0.30 \pm 0.02$; means, SD; RA: women: $0.72 \pm 0.15$, men: $0.66 \pm 0.18$; main effect of SEX; $p=0.004$ ). Activity of the five hours with lowest daily activity (L5) was significantly higher in men (861 \pm 572 ) than in women (594 \pm 607 ; main effect of SEX; $F_{1,72}=8.6$; $\left.p<0.05\right)$, and men also had earlier wake times than women (wake times men: $7.9 \mathrm{~h} \pm 0.7$; women $8.3 \mathrm{~h} \pm 0.8$; main effect of SEX; $\left.F_{1,72}=4.0 ; p<0.05\right)$, which did not result in any other statistical differences of sleep duration or time in bed between men and women. There were no significant main effects or interactions for the remaining circadian or sleep variables.

\section{Blood variables}

There was no difference between the two light exposure groups for any of the blood variables between in the pre- and the post-vaccination samples $(p>0.13$, Table 2$)$. In general, the CD4/CD8 ratio, C-reactive protein (CRP), leucocyte count and percentage of neutrophil lymphocytes were higher in the pre- than the post vaccination sample (main effect 
of SESSION; $p<0.05$; Table 2), while the absolute lymphocyte count and percentage were higher in the post- than the pre-vaccination sample $(p<0.05)$. For the younger subgroup (see statistics) of patients, the erythrocyte counts $(E C)$, haemoglobin $(H G)$, haematocrit $(H K)$, mean corpuscular haemoglobin concentrations $(\mathrm{MCHC}$ ) and lymphocytes (in \% from automated processing) were significantly higher than in the older patient group (main effect of $A G E ; p<0.015)$.

\section{Influenza vaccination response}

Pre- and post-vaccination antibody titers did not show significant differences between the light exposure groups $(p>0.07)$. However, the post/pre-vaccination ratios revealed a significantly higher increase in antibody titer for the $\mathrm{N} 3 \mathrm{H} 2$ virus strain in the high than the low light group (Table $3 a ; F_{1,70}=6.8 ; p=0.01$; Figure 1). On visual inspection, the IB post/prevaccination ratio seems similar to the $\mathrm{N} 3 \mathrm{H} 2$ but the difference did not reach significance. It revealed a trend $(p=0.08)$ for slightly higher antibody titer in the high light exposure group (for significant effects with AGE and SEX see Supplemental Results).

The geometric mean antibody titer (GMT) for pre- and post-vaccination responses are shown in Table $2 b$ for both light exposure groups. From a cohort perspective, the postvaccination seroconversion rates (i.e. the percentage of patients with a GMT greater or equal 40) were at least $75 \%$ (Supplemental Table S2). The seroprotection rates, which reflect a 4fold increase of the GMT was greater or equal to $34 \%$ for all three virus strains. When performing a survival analysis on the reverse cumulative distributions of GMTs with the low and high light patient group (Figure 2), there were no significant differences for the three influenza virus strains (Kaplan-Meier on log-ranked values; $p>0.4$ ).

\section{Discussion}

A group of dementia patients with long-term brighter daytime light exposure showed significantly greater circadian inter-daily stability and higher relative amplitude of circadian rest-activity cycles along with higher antibody production in response to the influenza virus strain $\mathrm{N} 3 \mathrm{H} 2$ than the patient group with lower bright light exposure. The seroconversion and seroprotection rates, which are standard criteria to evaluate the effectiveness of a vaccination within a cohort, showed that both light exposure groups were well protected against the influenza virus. However, in highly vulnerable patients, any additional benefit at the individual immunological response level is desirable. Even more so because the effects could have been indirectly conveyed via the improved circadian rest-activity cycles. Indeed, this assumption is corroborated by the significantly higher RA from rest-activity cycles in those participants with enhanced light exposure. A higher relative amplitude with greater inter-daily stability may have repercussions on other health related parameters, as shown for 
a variety of diseases (reviewed e.g. in ${ }^{44},{ }^{45}$ ). Although only one influenza strain showed significantly increased response, one other strain (IB) trended to increase in a similar way $(p=0.08)$.

The results from blood factors measured before and after the influenza vaccination were all in the normal range and revealed some vaccination effects (e.g. lymphocyte count) and some lower values for the older subgroup (e.g. erythrocytes and haemoglobin). There was no statistically significant difference in any blood factor between the light exposure groups, including those involved in T-cell related immune responses (e.g. the CD4 and CD8 cells and the CD4/CD8 ratio). Any differences in antibody responses were rather conveyed through the humoral and adaptive immunity driving cells, the B-lymphocytes.

More daytime light (= increased Zeitgeber strength) has led to increased amplitude for example of melatonin secretion profiles in older institutionalised individuals ${ }^{46}$, or stabilised rest-activity profiles in Alzheimer patients ${ }^{13}$. Therefore, a likely interpretation of the higher immune responses in our patient group with brighter light exposure is that these responses were transduced by overall higher circadian rhythm stability of central (and peripheral clocks). It is well known that stress, emotional or other biopsychological and social aspects (bidirectionally) affect acute and long-term immune responses ${ }^{47,}{ }^{48}$. Therefore, it may be that brighter light exposure improved immune responses not only by increasing circadian stability but also by the light-induced positive emotions such as improved mood, greater alertness and quality of life ${ }^{11}$.

Beneficial non-visual effects of brighter light exposure can obviously be best provided by natural daylight (with appropriate UV-protection of skin and eyes), but also by improved

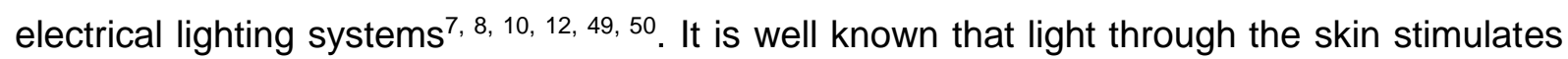
innate and adaptive immune responses, thus preventing different diseases through Vitamin D production from Ultraviolet B (UVB) solar radiation ${ }^{51,52}$. In our dementia patient cohort, we used wrist-worn light sensors which continuously measured illuminance. A limitation of our study may be that from these measures we could not disentangle whether participants were outside and exposed to direct sun (and UVB) or spending time inside, where window glazing absorbs UVB radiation.

In summary, there may be underestimated benefits from regular brighter and natural light exposures in older and frail individuals: better circadian entrainment, mood, rest-activity and immune functions. Our findings provide some evidence that in patients with dementia, long-term brighter light exposure may foster increased antibody titer production in response to the annual influenza vaccination, either directly or via circadian rhythm stabilisation. Further demonstration of positive effects of light to boost immune functions would open a whole new area of research with wide applications. There is a need to determine the optimal timing, duration and qualities of light required. 
In summary, we have first evidence that brighter daily illuminance levels, provided by natural daylight or improved electrical lighting, are beneficial for demented patients in modulating specific immune responses.

\section{Conflict of interest}

MM, AWJ, JLS and CC are members of the Daylight Academy. CC has had the following commercial interests in the last two years (2019-2020) related to lighting: honoraria, travel, accommodation and/or meals for invited keynote lectures, conference presentations or teaching from Toshiba Materials, Velux, Firalux, Lighting Europe, Electrosuisse, Novartis, Roche, Elite, Servier, and WIR Bank.

\section{Acknowledgments}

We thank all care givers and professionals of the Nursing home 'Das Heim', especially Michael Schmieder and Katharina Bieler, for without their professional help and support, the study would not have been possible. We thank Anne-Claire Sigrist and team of for great help with the antibody titer assays and Adriano Fontano for his initial advice with the immunological variables. We are also grateful to Markus Oetiker from Zumtobel Licht AG (Dornbirn, Austria) for help with programming the dynamic light settings. We also thank Timo Fuhrman with the data collection, light measurements and him and Daniel Hulliger for his valuable help with data processing. We also acknowledge Licht Zumtobel AG (Austria), Medica AG (Switzerland) and Camntech (UK) and Salimetrix (UK) for giving us a discount price on their products.

\section{Funding}

This study was generously supported by the Velux Foundation Switzerland (Proposal 259a: Post-doctoral Fellowship), the Age Stiftung Switzerland (I-2010-015) and Sonnweid Stiftung, Switzerland. 


\section{Tables and Figures}

Table 1

\begin{tabular}{l|cccc} 
Variable & $\begin{array}{c}\text { Low Light } \\
\text { Exposure Group }\end{array}$ & SD & $\begin{array}{c}\text { High Light } \\
\text { Expsoure Group }\end{array}$ & SD \\
\hline IS * & 0.33 & $(0.15)$ & 0.38 & $(0.14)$ \\
IV & 1.05 & $(0.30)$ & 1.18 & $(0.38)$ \\
L5 & 705.77 & $(591.84)$ & 655.15 & $(625.49)$ \\
M10 & 3969.53 & $(3263.18)$ & 4241.26 & $(3756.49)$ \\
RA * & 0.66 & $(0.18)$ & 0.72 & $(0.15)$ \\
BT (h) & 19.50 & $(1.00)$ & 19.57 & $(1.31)$ \\
WT (h) & 8.24 & $(0.71)$ & 8.10 & $(0.79)$ \\
TIB (h) & 12.71 & $(1.25)$ & 12.52 & $(1.73)$ \\
Wake (h) & 2.14 & $(1.26)$ & 1.84 & $(1.09)$ \\
Sleep duration (h) & 10.28 & $(2.26)$ & 10.40 & $(2.48)$ \\
SE (\%) & 80.09 & $(12.08)$ & 82.23 & $(10.90)$ \\
Fragmentation Index & 50.81 & $(24.87)$ & 46.69 & $(18.86)$
\end{tabular}

\section{Table 2:}

Circadian and sleep variables (derived from activity monitors) for low and high light exposure (low LE, high LE) groups (mean, SD in brackets; $\mathrm{n}=80$ ). IS = Inter-daily stability; IV = intradaily variability; L5 = 5 hours with lowest activity; M10 10 hours with highest activity; RA = relative amplitude (see Ref. ${ }^{42}$ for more details; BT = habitual bedtime (h); WT = habitual waketime (h); TIB = Time in bed (h); Wake = waketime during scheduled sleep (h); Sleep duration (h); SE = sleep efficiency (\%); sleep time / TIB x 100); Fragmentation index (dimensionless). ${ }^{*}=p<0.05$ between light exposure groups. 
Table 2

Blood Marker

CD4 (count $/ \mu \mathrm{l})$

CD8 (count $/ \mu \mathrm{l})$

CD4/CD8 Ratio *

Lymphocytes (count $/ \mu \mathrm{l})$ *

$\operatorname{CRP}(\mathrm{mg} / \mathrm{l})$ *

LC (count $/ \mu \mathrm{l})$ *

EC (G/I)

HB (g/l)

HK (I/I)

$\operatorname{MCV}(\mathbf{f l})$

$\mathrm{MCH}$ (pg)

$\mathrm{MCHC}(\mathrm{g} / \mathrm{l})$

TC (G/I)

Neutrophil (\%) *

Eosinophil (\%)

Basophil (\%)

Monocytes (\%)

Lymphocytes (\%) *

LUC (\%)

FACS (count $/ \mu \mathrm{l})$
Pre-vaccination (SEM)

879.23

435.74

(33.17)

(35.63)

2.76

(0.19)

(86.17)

1775.00

10.40

7110.00

(243.31)

4.37

130.09

0.40

(0.005)

90.64

(0.52)

29.77

$(0.19)$

328.40

292.91

62.71

3.66

0.53

(0.94)

23.94

(0.27)

(57.60)

6.07

3.08

1331.20
Post-vaccination (SEM)

892.61

(36.09)

448.29

(33.43)

2.65

(0.18)

1881.01

(72.40)

6.59

6602.50

(189.08)

4.32

128.85

0.39

(0.004)

90.94

29.85

(0.16)

328.43

(0.78)

284.41

59.92

4.12

0.54

(55.55)

\section{Table 2:}

Blood analyses before and 6 weeks after the influenza vaccination. $\mathrm{CRP}=\mathrm{C}$-reactive protein, $\mathrm{LC}=$ leucocytes, $\mathrm{EC}=$ erythrocytes, $\mathrm{HB}=$ haemoglobin, $\mathrm{HK}=$ haematocrit, $\mathrm{MCV}=$ mean corpuscular volume, $\mathrm{MCH}=$ mean corpuscular haemoglobin, $\mathrm{MCHC}=$ mean corpuscular haemoglobin concentration, TC = thrombocytes, LUC = large unstained cells, FACS $=$ fluorescence activated cell sorting (CD3, CD4, CD8); $n=80 ;{ }^{*}=p<0.05$; main difference between pre- and post-vaccine session. 
Table 3a

\begin{tabular}{c|cccc}
\multicolumn{1}{c}{ Titer } & $\begin{array}{c}\text { Low Light } \\
\text { Exposure Group }\end{array}$ & \multicolumn{3}{c}{$\begin{array}{c}\text { High Light } \\
\text { Exposure Group }\end{array}$} \\
\hline Pre (H3N2) & 140.8 & $(12.3)$ & 150.3 & $(25.3)$ \\
Post (H3N2) & 421.3 & $(69.2)$ & 627.5 & $(146.3)$ \\
Ratio (H3N2) * & 3.4 & $(0.6)$ & 9.9 & $(3.2)$ \\
Pre (H1N1) & 39.5 & $(8.4)$ & 62.5 & $(12.3)$ \\
Post (H1N1) & 477.5 & $(93.8)$ & 401.6 & $(112.6)$ \\
Ratio (H1N1) & 31.1 & $(7.9)$ & 26.7 & $(9.4)$ \\
Pre (IB) & 65.4 & $(9.4)$ & 56.0 & $(9.4)$ \\
Post (IB) & 232.6 & $(61.2)$ & 201.9 & $(29.5)$ \\
Ratio (IB) & 5.6 & $(1.6)$ & 9.7 & $(2.4)$
\end{tabular}

Table 3a: Absolute influenza antibody titers pre- and post-vaccination for three virus strains: H3N2 ( $n=78), H 1 N 1$ ( $n=79)$ and IB ( $n=80)$ as well as ratio (post/pre) for the low and high light group); means and (SEM). ${ }^{*}=$ significant differences between ratio of the low and the high light exposure group $(p<0.05)$. 


\section{Figure 1}

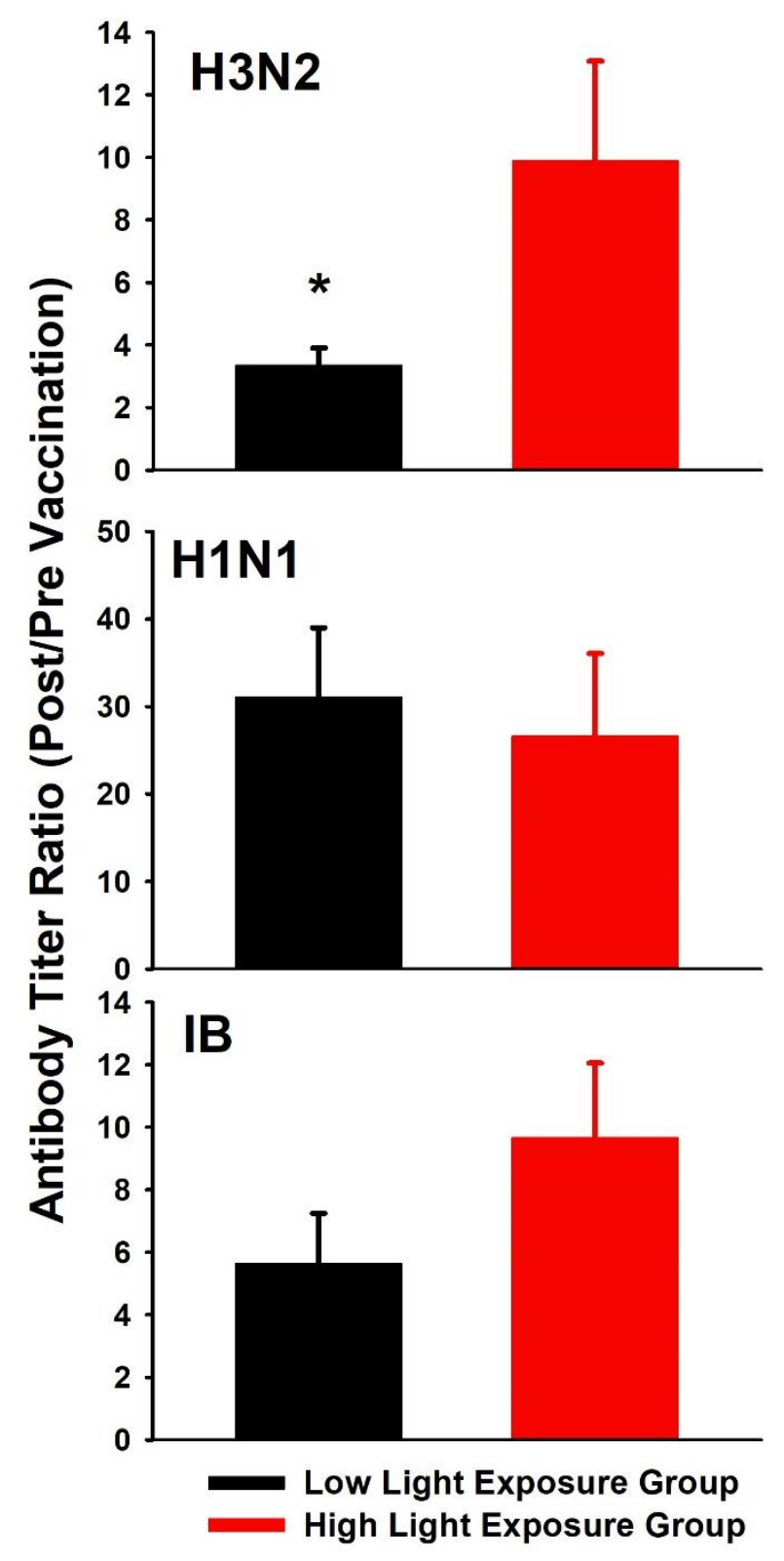

\section{Figure 1}

Mean values (+ SEM) for antibody titer ratios (post-vaccination/pre-vaccination) for all three influenza strains and both sub-groups of patients [high (red bars) vs. low light exposure group (black bars)]: H3N2 ( $n=78)$; H1N1 $(n=79)$; IB $(n=80) .{ }^{*}=p<0.05$. 


\section{Figure 2}

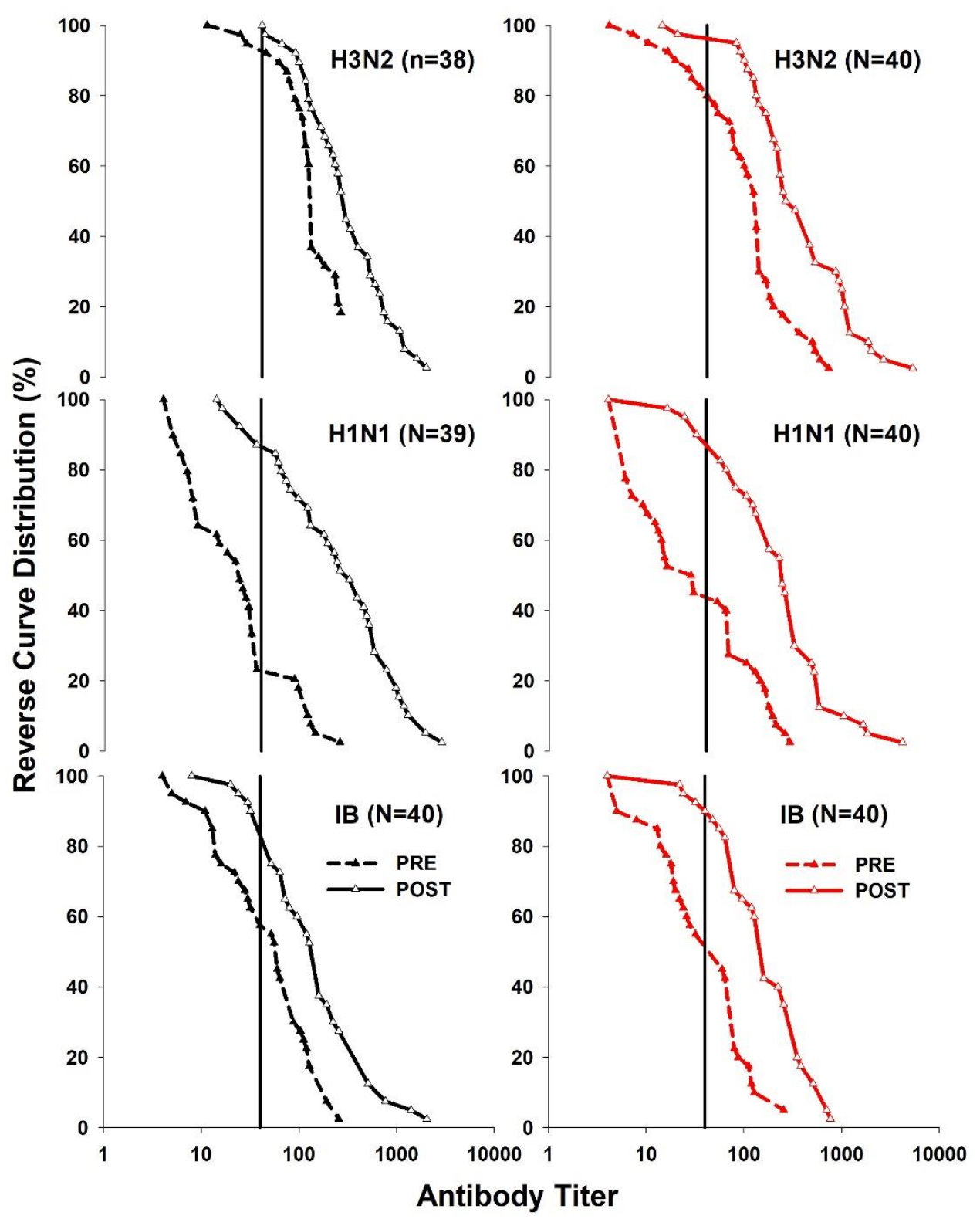

\section{Figure 2}

Reverse curve distribution plots from geometric mean antibody titers (GMT) for prevaccination (dashed lines) and post-vaccination (solid lines) samples and the three influenza virus strains (H3N2, upper graph; H1N1, middle graph; IB, lower graph). The data is expressed in percentage for both light exposure groups of patients separately (left panel and black symbols = low light exposure group; right panel and red symbols = high light exposure group; open triangles and dashed lines = pre-vaccination antibody titers; filled triangles and solid lines = post-vaccination antibody titers). The vertical line in each graph represents the threshold for GMT titers of seroprotection by the influenza vaccination (i.e. a GMT $\geq 40$ ). 
bioRxiv preprint doi: https://doi.org/10.1101/2020.11.30.405175; this version posted December 2, 2020. The copyright holder for this preprint (which was not certified by peer review) is the author/funder. All rights reserved. No reuse allowed without permission.

\section{References}

1. Wennberg AMV, Wu MN, Rosenberg PB, et al. Sleep disturbance, cognitive decline, and dementia: A review. Semin Neurol 2017;37:395-406.

2. Lee HB, Lyketsos CG. Depression in Alzheimer's disease: heterogeneity and related issues. Biol Psych 2003;54:353-362.

3. Swaab D, Dubelaar E, Hofman M, et al. Brain aging and Alzheimer's disease; use it or lose it. Prog. Brain Res 2002;138:343-373.

4. Musiek ES, Bhimasani M, Zangrilli MA, et al. Circadian rest-activity pattern changes in aging and preclinical Alzheimer disease. JAMA Neurol 2018;75:582-590.

5. Van Erum J, Van Dam D, De Deyn PP. Sleep and Alzheimer's disease: A pivotal role for the suprachiasmatic nucleus. Sleep Med Rev 2018;40:17-27.

6. Roccaro I, Smirni D. Fiat Lux: The light became therapy. An overview on the bright light therapy in alzheimer's Disease sleep disorders. J Alzheimers Dis 2020;77:113-125.

7. Ancoli-Israel S, Gehrman P, Martin J, et al. Increased light exposure consolidates sleep and strengthens circadian rhythms in severe alzheimer's disease patients. Behav Sleep Med 2003;1:22-36.

8. Wahnschaffe A, Nowozin C, Haedel S, et al. Implementation of dynamic lighting in a nursing home: impact on agitation but not on rest-activity patterns. Curr Alzheimer Res 2017;14:10761083.

9. Mishima K, Hishikawa Y, Okawa M. Randomized, dim light controlled, crossover test of morning bright light therapy for rest-activity rhythm disorders in patients with vascular dementia and dementia of alzheimer`s type. Chronobiol Int 1998;15:647-654.

10. Figueiro M, Plitnick B, Roohan C, et al. Effects of a tailored lighting intervention on sleep quality, rest-activity, mood, and behavior in older adults with Alzheimer disease and related dementias: A randomized clinical trial. Clin Sleep Med 2019;15:1757-1767.

11. Münch M, Schmieder M, Bieler K, et al. Bright light delights: effects of daily light exposure on emotions, rest-activity cycles, sleep and melatonin secretion in severely demented patients. Curr Alzheimer Res 2017;14:1063-1075.

12. Riemersma-van der Lek RF, Swaab DF, Twisk J, et al. Effect of bright light and melatonin on cognitive and noncognitive function in elderly residents of group care facilities: a randomized controlled trial. JAMA 2008;299:2642-2655.

13. Van Someren EJW, Kessler A, Mirmiran M, et al. Indirect bright light improves circadian rest-activity rhythm disturbances in demented patients. Biol Psych 1997;41:955-963.

14. Sloane PD, Williams CS, Mitchell CM, et al. High-intensity environmental light in dementia: effect on sleep and activity. J Am Geriatr Soc 2007;55:1524-1533.

15. Hjetland GJ, Pallesen S, Thun E, et al. Light interventions and sleep, circadian, behavioral, and psychological disturbances in dementia: A systematic review of methods and outcomes. Sleep Med Rev 2020;52:101310.

16. Fontana Gasio P, Kräuchi K, Cajochen C, et al. Dawn-dusk simulation light therapy of disturbed circadian rest-activity cycles in demented elderly. Exp Gerontol 2003;38:207-216.

17. Bromundt V, Wirz-Justice A, Boutellier M, et al. Effects of a dawn-dusk simulation on circadian rest-activity cycles, sleep, mood and well-being in dementia patients. Exp Gerontol 2019;124:110641.

18. Thompson W, Shay D, Weintraub E, et al. Mortality associated with influenza and respiratory syncytial virus in the United States. JAMA 2003;289:179-86.

19. Weinberger B, Herndler-Brandstetter D, Schwanninger A, et al. Vaccines: Biology of Immune Responses to Vaccines in Elderly Persons. Clin Infect Dis 2008;46:1078-1084.

20. Goodwin K, Viboud C, Simonsen L. Antibody response to influenza vaccination in the elderly: A quantitative review. Vaccine 2006;24:1159-1169.

21. Frasca D, Blomberg B. B cell function and influenza vaccine responses in healthy aging and disease. Curr Opin Immunol 2014;29:112-118.

22. Henry C, Zheng NY, Huang M, et al. Influenza virus vaccination elicits poorly adapted B cell responses in elderly individuals. Cell Host Microbe 2019;25:357-366.e6.

23. Grubeck-Loebenstein B, Wick G. The aging of the immune system. Advanc Immunol 2002; Volume 80:243-284. 
bioRxiv preprint doi: https://doi.org/10.1101/2020.11.30.405175; this version posted December 2, 2020. The copyright holder for this preprint (which was not certified by peer review) is the author/funder. All rights reserved. No reuse allowed without permission.

24. Monto A, Ansaldi F, Aspinall R, et al. Influenza control in the 21st century: Optimizing protection of older adults. Vaccine 2009;27:5043-53.

25. Kim SW, Su K. Using psychoneuroimmunity against COVID-19. Brain Behav Immun 2020;87:4-5.

26. Chidambaram P. State reporting of cases and deaths due to COVID-19 in long-term care facilities. Coronavirus (COVID-19): Kaiser Family Foundation, (KFF), 2020.

27. Blanco-Tarrio E, Blanco Sánchez G. Primary care, residential homes for the elderly, and COVID-19. Semergen 2020;46 Suppl 1:26-34.

28. Fisman DN, Bogoch I, Lapointe-Shaw L, et al. Risk factors associated with mortality among residents with coronavirus disease 2019 (COVID-19) in long-term care facilities in Ontario, Canada. JAMA Netw Open 2020;3:e2015957.

29. Besedovsky L, Lange T, Haack M. The sleep-immune crosstalk in health and disease. Physiol Rev 2019;99:1325-1380.

30. Irwin M, Opp M. Sleep health: Reciprocal regulation of sleep and innate immunity. Neuropsychopharmacology 2017;42:129-155.

31. Haspel J, Anafi R, Brown M, et al. Perfect timing: circadian rhythms, sleep, and immunity an NIH workshop summary. JCI insight 2020;5:e131487.

32. Born J, Lange T, Hansen K, et al. Effects of sleep and circadian rhythm on human circulating immune cells. J Immunol 1997;158:4454-64.

33. Cermakian N, Lange T, Golombek D, et al. Crosstalk between the circadian clock circuitry and the immune system. Chronobiol Int 2013;30:870-88.

34. Scheiermann C, Gibbs J, Ince L, et al. Clocking in to immunity. Nat Rev Immunol 2018; 18:423-437.

35. Labrecque N, Cermakian N. Circadian clocks in the immune system. J Biol Rhythms 2015;30:277-90.

36. Hayashi O, Kikuchi M. The influence of phase shift in the light-dark cycle on humoral immune responses of mice to sheep red blood cells and polyvinylpyrrolidone. J Immunol 1985;134:1455-61.

37. Abele SH, Meadows KE, Medeiros D, et al. Time is on the immune system's side, yes it is. Yale J Biol Med 2019;92:225-231.

38. Inokawa $\mathrm{H}$, Umemura $\mathrm{Y}$, Shimba $\mathrm{A}$, et al. Chronic circadian misalignment accelerates immune senescence and abbreviates lifespan in mice. Sci Rep 2020;10:2569.

39. Roberts JE. Light and Immunomodulation. Ann N. Y. Acad.Sci 2000;917:435-445.

40. Park S-J, Tokura H. Bright Light Exposure During the Daytime Affects Circadian Rhythms of Urinary Melatonin and Salivary Immunoglobulin A. Chronobiol Int 1999;16:359 - 371.

41. Harrell L, Marson D, Chatterjee A, et al. The Severe Mini-Mental State Examination: a new neuropsychologic instrument for the bedside assessment of severely impaired patients with Alzheimer disease. Alzheimer Dis Assoc Disord 2000;14:168-75.

42. Van Someren EJW, Swaab DF, Colenda CC, et al. Bright light therapy: improved sensitivity to its effects on rest-activity rhythms in Alzheimer patients by application of nonparametric methods. Chronobiol Int 1999;16:505-18.

43. Gabay C, Bel M, Combescure C, et al. Impact of synthetic and biologic disease-modifying antirheumatic drugs on antibody responses to the AS03-adjuvanted pandemic influenza vaccine: a prospective, open-label, parallel-cohort, single-center study. Arthritis Rheum 2011;63:1486-96.

44. Abbott SM, Zee PC. Circadian rhythms: implications for health and disease. Neurol Clin 2019;37:601-613.

45. Leng $\mathrm{Y}$, Musiek ES, $\mathrm{Hu} \mathrm{K}$, et al. Association between circadian rhythms and neurodegenerative diseases. Lancet Neurol 2019;18:307-318.

46. Mishima K, Okawa M, Shimitzu T, et al. Diminished melatonin secretion in the elderly caused by insufficient environmental illumination. J Clin Endocrinol Metab 2001;86:129-134.

47. Vedhara K, Cox N, Wilcock G, et al. Chronic stress in elderly carers of dementia patients and antibody response to influenza vaccination. Lancet 1999;353:627-31.

48. Dhabhar FS. Effects of stress on immune function: the good, the bad, and the beautiful. Immunol Res 2014;58:193-210. 
49. Dowling G, Mastick J, Hubbard E, et al. Effect of timed bright light treatment for rest-activity disruption in institutionalized patients with Alzheimer's disease. Int J Geriatr Psychiatry 2005;20:738-43.

50. Mishima K, Okawa M, Hishikawa Y, et al. Morning bright light therapy for sleep and behavior disorders in elderly patients with dementia. Acta Psychiatr Scand 1994;89:1-7.

51. Hawk J. Safe, mild ultraviolet-B exposure: An essential human requirement for vitamin $D$ and other vital bodily parameter adequacy: A review. Photodermatol Photoimmunol Photomed 2020.

52. Reichrath J. Lessons learned from Paleolithic models and evolution for human health: A snap shot on beneficial effects and risks of solar radiation. Adv Exp Med Biol 2020;1268:3-15. 